\title{
Research in the heat transfer of water in a bathtub of different shapes Qi Sun ${ }^{1, a}$ \\ ${ }^{1}$ North China Electric Power University, Baoding 071000, China; \\ A1335886716@qq.com
}

Keywords: Equivalent Size, Finite Element Analysis, Convection Heat Transfer, Numerical Simulation.

\begin{abstract}
The article established mathematical models to gain the temperature field of water in a bathtub of different shapes. At the beginning, the basic model including heating and cooling model is established on condition that the bathtub is $1.8 \times 0.7 \times 0.8$ cuboid without a person in it. Afterwards, the shape and the volume of the bathtub are taken into consideration. the equivalent size is introduced to establish the relationship of equation between different shapes of the bathtub. The result shows that different shapes do not make influences on the division of temperature areas in the bathtub but affect the heat transfer rate of water heating and cooling Meanwhile, the result is performed by means of finite element analysis, using the software Fluent[1].
\end{abstract}

\section{Introduction}

In the previous research, J. X. Zhang did some researches on the influence for swimming pool water temperature field in different heating modes. One of the modes is traditional heating mode, the other is called floor heating mode. Results show that in the same conditions, the swimming pool's temperature field with floor heating mode is more homogeneous than the traditional heating mode, time to achieve the stable temperature field is shorter[2]. In our paper, the bathtub is heated by means of the hot water from the faucet.

Most of the these references contribute a lot to the problem we are analyzing. Nevertheless, little has been devoted to the heat transfer in bathtub., not to mention the influence of the shape and size of the tub.

\section{Analysis of the Problem}

The basic model including heating and cooling model is established on condition that the bathtub is 1.8 $\times 0.7 \times 0.8$ cuboid without a person in it. In this problem, the shape and volume of the bathtub. Thus, we divide the problem into two sections. The section one introduced the equivalent size to establish the relationship of equation between different shapes of the bathtub. In the section two, simulations are made to verity the result.

\section{Established Model Considering Bathtub’s Shape and Volume}

In this section, considering the complex boundary conditions are not able to be dealt with in the basic model, the equivalent size is introduced to establish the relationship of equation between different shapes of the bathtub. In order to make the model applied more extensively, it is necessary to make the equations of heat transfer and boundary conditions as close as possible to the initial model. According to the fluid mechanics and heat transfer theory, the closer the equivalent sizes of the two shapes are, the more similar the equations of heat transfer and boundary conditions are. So we regard the equation of the equivalent size as a constraint in the programming problem.

Considering that the shape of the bathtub researched in the basic model is cuboid, we try to convert other shapes to the cuboid as possible as we can according to some criterions which include the equation of the equivalent size, volume and inner surface area.

Here the equivalent size is defined as $l=A / \chi$, where $A$ represents the value of area and $\chi$ indicates the perimeter of the shape. Based on it, a ternary two-degree equation set is built. Due to the diverse solutions to the equation set, we build a non-linear programming model in which the difference of 
surface area between the shape and the converted one is regarded as the object. It is presented as follows:

$$
\begin{aligned}
& \min =[2 \times(B \cdot H+H \cdot L)+B \cdot L-\oiint f(x, y, z) d S] \\
& s t\left\{\begin{array}{l}
\frac{\iint f\left(x, y, \frac{H}{2}\right) d \sigma}{\oint f\left(x, y, \frac{H}{2}\right) d s}=\frac{L \cdot B}{2(L+B)} \\
\int f\left(x, y, \frac{H}{2}\right) d x d y \cdot H=B \cdot H \cdot L \\
\iint f\left(\frac{L}{2}, y, z\right) d \sigma \\
\frac{\oint f\left(\frac{L}{2}, y, z\right) d s}{2(H+B)}
\end{array}\right.
\end{aligned}
$$

Where $\frac{\iint f\left(x, y, \frac{H}{2}\right) d \sigma}{\oint f\left(x, y, \frac{H}{2}\right) d s}=\frac{L \cdot B}{2(L+B)}$ is established based on the heating process in direction of the bathtub's length.

$$
\text { Where } \frac{\iint f\left(\frac{L}{2}, y, z\right) d \sigma}{\oint f\left(\frac{L}{2}, y, z\right) d s}=\frac{B \cdot H}{2(H+B)} \text { is established based on the cooling process in direction of the }
$$
bathtub’s height.

Take the elliptic column as an example. Based on the thought of equivalent size, we utilize the programming model to convert the elliptic column into a cuboid. Then do some simulation in terms of the original and transformed shape respectively for contrast.

We simulate the temperature field [3] in both cooling and heating process and take the condition after five minutes as an example to present in Fig 1 and Fig 2.

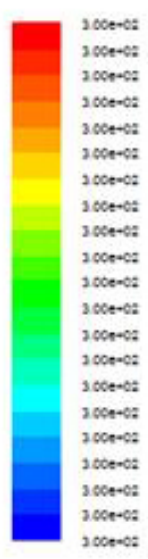

\section{Cooling Process}
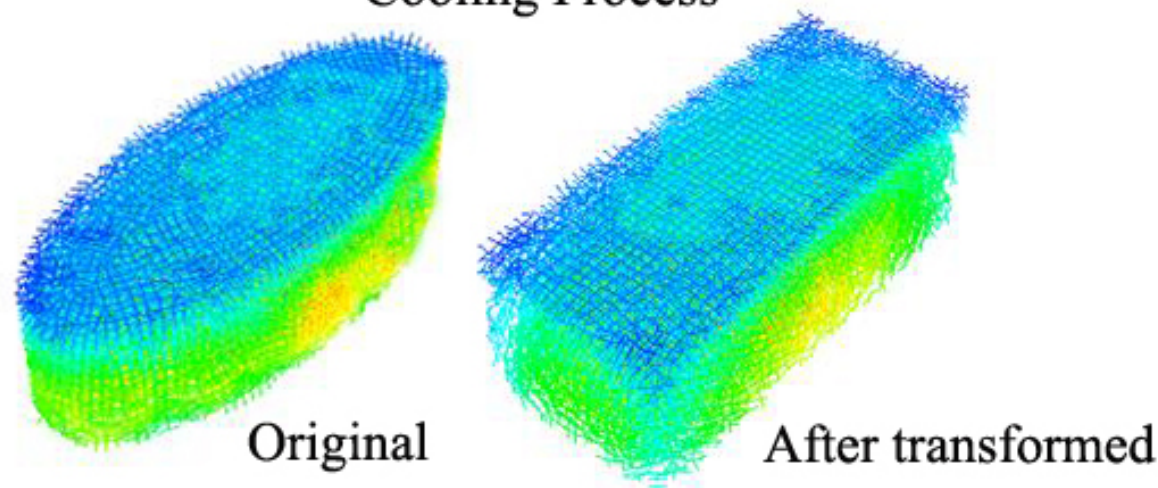

Fig 1 the simulation of the original and transformed shape in cooling process 

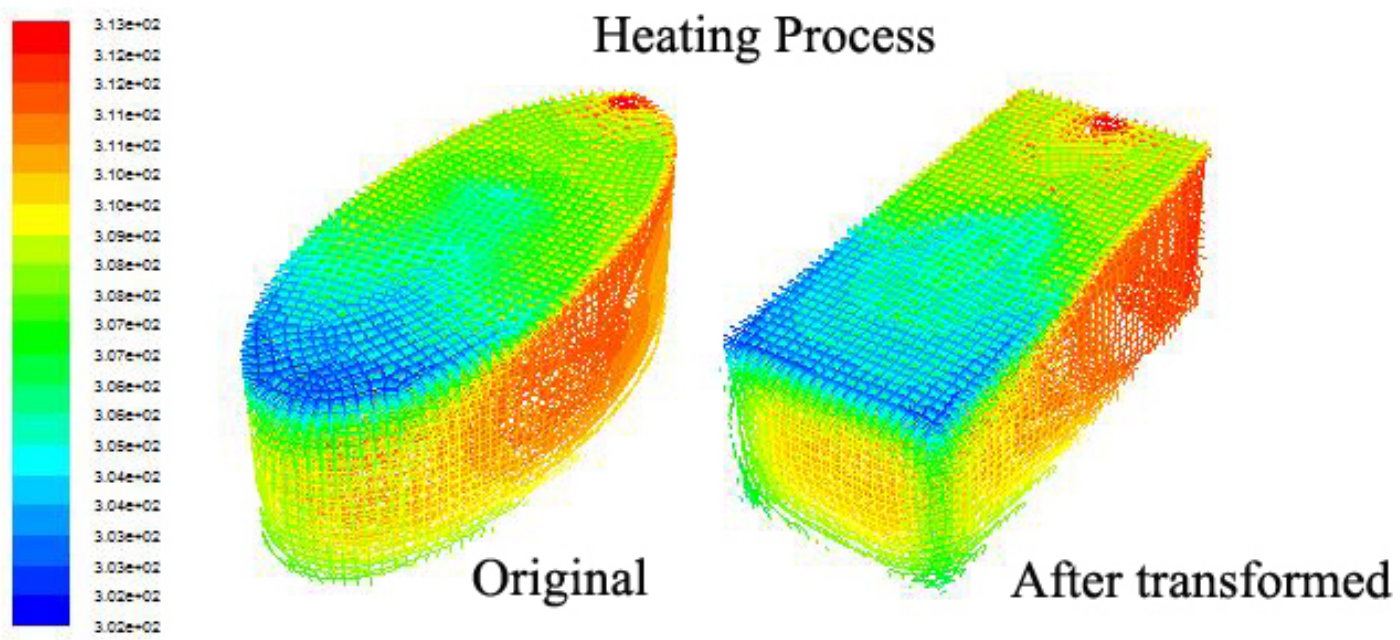

Fig 2 the simulation of the original and transformed shape in heating process

As is shown above, it is obvious that the temperature distributions of the original and transformed shape are very similar at the same moment. It is demonstrated that the programming model based on the equivalent size is effective.

Meanwhile, we work out the change curve of the temperature and positions which are selected from the neutral surface. The results are shown as follows:
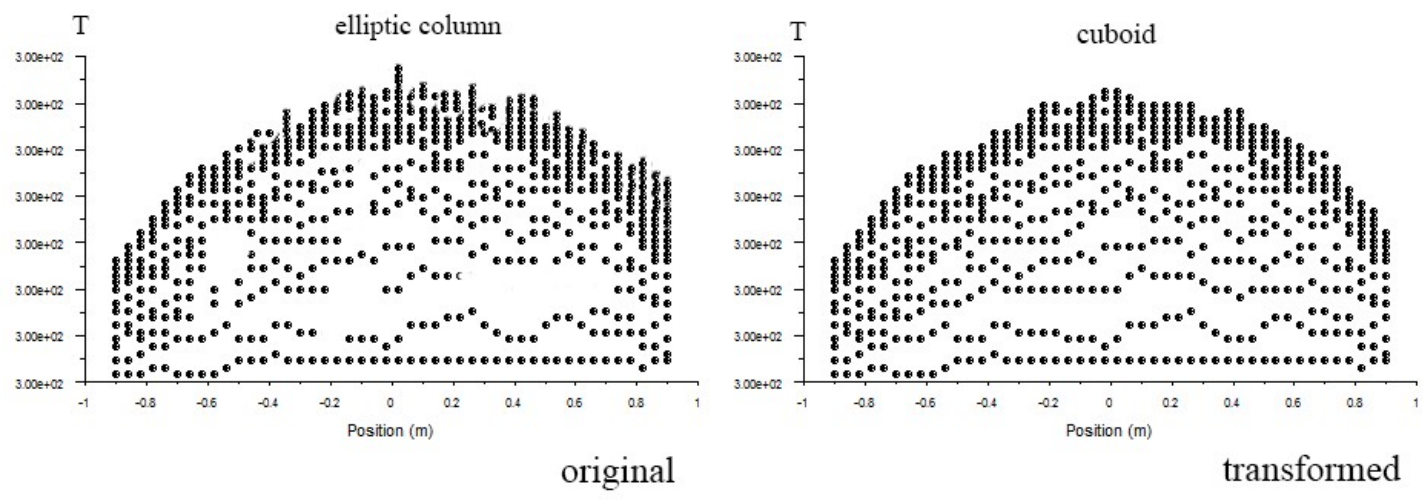

Fig 3 the change curve of the temperature in cooling process
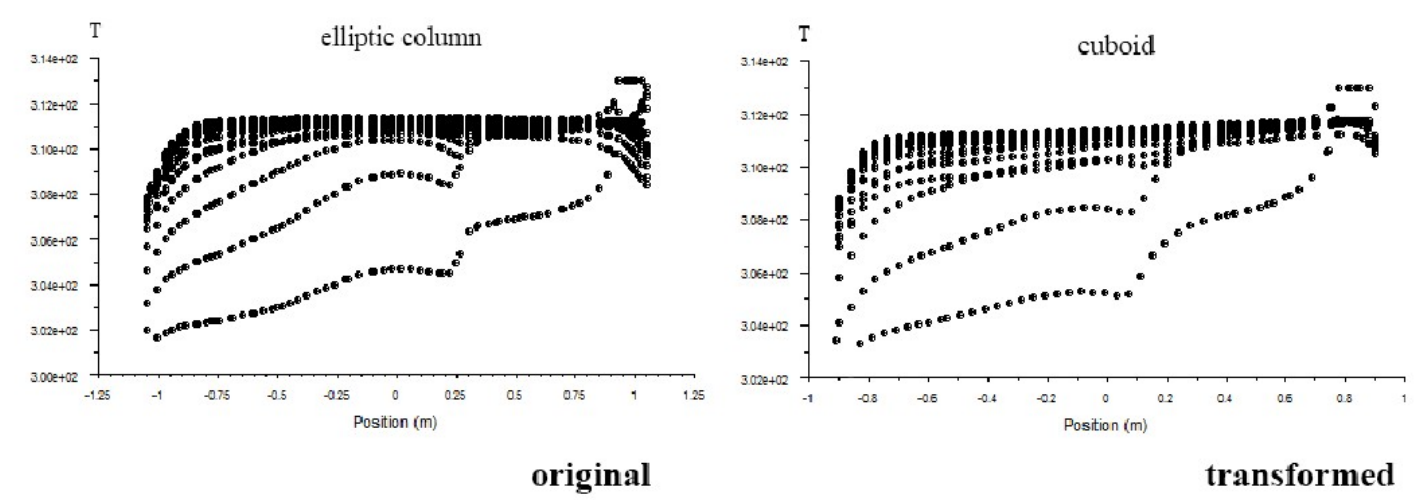

Fig 4 the change curve of the temperature in heating process

From the figure above we can see that the change trends of the temperature maintain in accordance on the neutral surface of the original and transformed shape. 


\section{Summary}

In this paper, the model is built by taking the influences of the shape, volume of the bathtub into account. Meanwhile, we introduce the equivalent size to simplify the model. We establish the non-linear programming model to research the difference of various shapes and the result presents that the elliptic column is the best shape to choose.

\section{References}

[1] ZHANG Shujia, LI Xianhua, ZHU Baolin, ”Applicability of k- $\varepsilon$ Eddy Viscosity Turbulence Models on Numerical Simulation of Centrifugal Pump", Journal of Mechanical Enginering,vol.45,No.4,pp.238-242,April 2009.

[2] Zhang Jinxia, 2014, “The Simulation Analysis of the Influence for Swimming Pool Water Temperature Field in Different Heating Modes”,PhD diss., Yanshan University., 2014.

[3] Zhou Wei, Wang Xiaochan, "Unsteady Temperature Simulation under Variable Boundary Conditions for Venlo Type Conditions”, Transaction of the Chinese Society of Agricultural Machinery, vol.45, No11, ,pp.305-307, November, 2014. 